\title{
Strategy to Replace Animal-Derived ECM by a Modular and Highly Defined Matrix
}

\author{
Yevheniia Nesterenko 1,3\#, Xenia Dolde2,3\#, Marcel Leist2,4 and Olga Mayans ${ }^{1}$ \\ ${ }^{1}$ Biophysics and Structural Biology, University of Konstanz, Konstanz, Germany; ${ }^{2}$ In vitro Toxicology and Biomedicine, Dept inaugurated by \\ the Doerenkamp-Zbinden Foundation, University of Konstanz, Konstanz, Germany; ${ }^{3}$ Konstanz Research School Chemical Biology (KoRS-CB), \\ University of Konstanz, Konstanz, Germany; ${ }^{4}$ CAAT-Europe, University of Konstanz, Konstanz, Germany
}

\begin{abstract}
Many extracellular matrices (ECM) used for modern cell culture are derived from animals. An alternative approach is the recombinant production of individual matrix protein components. A further development of this strategy uses a constant core protein polymer that is modifiable with functional domains of various ECM proteins. This way, a single, highlydefined ECM system could be used for a large variety of cell types. Self-assembling protein domains from human muscle sarcomeres, termed ZT material (ZT), have been shown to be suitable for this modular approach of generating ECMs. We explored in a proof-of-concept study whether $Z T$ modified with the fibronectin 10 domain $\left(Z T^{F n} 10\right)$ is able to substitute bovine serum-derived fibronectin as a coating for neural crest cell (NCC)-based toxicity testing. Human NCCs were generated from pluripotent stem cells and used in the automated version of an NCC migration assay (cMINC). ZTFn 10, but not the unmodified core material (ZT), allowed high migration activity. The classical CMINC setup, with bovine fibronectin coating, was used as positive control, and detailed analysis of NCC migration by time-lapse recording indicated that the novel ECM fully matched the bioactivity of the traditional ECM. A final set of experiments showed that various positive controls of the CMINC assay (PCB 180, LiCl, cytochalasin D) showed nearly identical inhibition curves on the traditional and the novel ECM. Thus, the CMINC, and possibly other bioassays, can be performed with a ZT-based ECM instead of traditional animal-derived protein coatings.
\end{abstract}

\section{Introduction}

While cell culture experiments have successfully replaced several animal studies, many in vitro methods still use animal-derived materials. The search for chemically-defined and non-animal sourced cell culture material has become a research priority in its own right. The goal is to make in vitro systems more reproducible (independent of lot effects) and to avoid ethically problematic procedures currently used to produce cell culture medium supplements, extracellular matrix, and analytical tools such as animal-derived antibodies.
Fetal bovine serum (FBS) is an example of an animal-derived product used commonly as a cell culture supplement. The unknown composition of FBS and its high batch-to-batch variation make it difficult to reproduce in vitro experiments between different laboratories (van der Valk et al., 2018). So far, serumfree media are in routine use only for a few cell types, such as LUHMES cells (Scholz et al., 2011) or induced pluripotent stem cells (iPSCs) (Hackland et al., 2017; Reichman et al., 2017; Sung et al., 2019), but no universal, chemically-defined, serum-free culture medium is available (van der Valk et al., 2018). The FCSfree database ${ }^{1}$ was launched to increase the use of serum-free

\footnotetext{
1 http://fcs-free.org/

\# contributed equally

Received March 18, 2020; Accepted May 28, 2020; Epub June 4, 2020; @ The Authors, 2020.

ALTEX 37(3), 482-489. doi:10.14573/altex.1912041

Correspondence: Olga Mayans, PhD

Biophysics and Structural Biology, University of Konstanz, Konstanz, Germany

University of Konstanz,Universitaetsstr. 10, 78457 Konstanz, Germany

(olga.mayans@uni-konstanz.de)

Marcel Leist, PhD

In vitro Toxicology and Biomedicine, Dept inaugurated by the Doerenkamp-Zbinden

Foundation at the University of Konstanz, Konstanz, Germany

University of Konstanz, Universitaetsstr. 10, 78457 Konstanz, Germany

(marcel.leist@uni-konstanz.de)
}

This is an Open Access article distributed under the terms of the Creative Commons Attribution 4.0 International license (http://creativecommons.org/licenses/by/4.0/), which permits unrestricted use, distribution and reproduction in any medium, provided the original work is appropriately cited. 
media by providing information on commercially available serum-free media for several cell types.

Another group of animal-derived cell culture reagents is formed by extracellular matrix (ECM) components, such as the widely used Matrigel ${ }^{\mathrm{TM}}$ and plasma fibronectin. Matrigel ${ }^{\mathrm{TM}}$ is a heterogeneous glycoprotein mixture extracted from tumors grown in mice. Its complex composition suffers from high intrinsic variability, limiting experimental control and leading to data inconsistencies (Hughes et al., 2010). Plasma fibronectin (Fn) is a $250 \mathrm{kDa}$ glycoprotein that assembles into dimers and consists of repetitive domains: 12 type I units, two type II units, and 1517 type III units (Pankov and Yamada, 2002). The $10^{\text {th }}$ Fn type III repeat domain $\left(\mathrm{Fn}^{10}\right)$ is responsible for the integrin receptor binding of fibronectin and, thereby, its cell attachment (Chi-Rosso et al., 1997). Specifically, cell attachment to $\mathrm{Fn}^{10}$ is mediated by the short peptide motif Arg-Gly-Asp (RGD). Due to its low recombinant yield and the complexity of its composition and assembly, most of the commercially available Fn for in vitro use is extracted from animal plasma (Oredsson et al., 2019). Consequently, Fn preparations are likely to contain other undefined plasma proteins as well.

Migration of neural crest cells (NCCs) is an important process in fetal development. During neurulation, NCCs delaminate from the dorsal part of the neural tube and migrate to target sites in the periphery where they differentiate into different cell types (LaBonne and Bronner-Fraser, 1999). Disturbed NCC migration can lead to severe malformations and disorders such as Hirschsprung's disease (Farlie et al., 2004). The migration of NCC is well studied in animals (Fuller et al., 2002; Bergeron et al., 2013; Usami et al., 2016), but few human in vitro data are available.

The migration inhibition of neural crest cell (MINC) assay was developed to evaluate developmental toxicants and signaling pathways (Zimmer et al., 2012). A high-throughput version of this in vitro assay, called circular migration inhibition of neural crest cell (cMINC) assay (Nyffeler et al., 2017b), is based on circular stoppers, which create a cell-free area around which cells are seeded; migration is initiated by removing the stoppers. The assay uses human NCCs generated from pluripotent stem cells to assess disturbances of their migration during fetal development (Nyffeler et al., 2017a). This migration requires extension of protrusions at the leading edge of the cell and disconnection from the ECM at the trailing edge (Conway and Jacquemet, 2019; Ridley et al., 2003). Thus, cell migration requires a constant switch between strong and weak attachment of the cell to the ECM as new adhesions are formed at the leading edge and old cell-ECM adhesions are disassembled (Shafqat-Abbasi et al., 2016).

NCC migration requires, therefore, dynamic interactions of surface integrin receptors with ECM proteins, and Fn is one of the key ECM proteins that facilitate NCC migration (Testaz et al., 1999; Henderson and Copp, 1997). Fn binds NCCs through $\alpha v \beta 1$ and $\alpha 8 \beta 1$ integrins and allows cell migration based on $\alpha 4 \beta 1$, $\alpha v \beta 3$, and $\alpha 8 \beta 1$ integrins (Perris et al., 1989; Testaz et al., 1999). Therefore, cell culture dishes for cMINC assays need to be coated with Fn or related ECM proteins. Interestingly, a variety of engineered biomaterials of defined composition have now become available that have proven to be viable substrate alternatives for in vitro cell culture (Cai and Heilshorn, 2014; Loo et al., 2015; Hellmund and Koksch, 2019). Biomaterials of homogenous composition and high purity optimally meet requirements for experimental standardization. In addition, biomaterials made of recombinant proteins offer a significant cost reduction for high-throughput studies, thanks to the economy of production and scalable yields of bacterial overexpression. Such biomaterials could permit developing a strategy for the replacement of animal-derived Fn in high-throughput assays like the cMINC assay.

In this respect, we developed a functionalized biopolymer, termed ZT, formed by the self-assembly of two human proteins: telethonin (Tel) and the two N-terminal immunoglobulin domains from titin (Z1Z2) (Bruning et al., 2010; Nesterenko et al., 2019). The complexation of Tel and Z1Z2 occurs naturally in the Z-disc of human muscle sarcomeres. In this complex, Tel acts as a molecular glue, becoming "sandwiched" between two antiparallel Z1Z2 and forming a robust intermolecular $\beta$-sheet that spans the three components (Zou et al., 2006; Marino et al., 2006). The ZT polymer uses a Z1Z2-Z1Z2 fusion tandem $\left(Z_{1212}\right)$ that leads to the spontaneous, propagative self-assembly of Z1Z2:Tel complexes into a polymer (Bruning et al., 2010). The $Z T$ polymer can be readily functionalized by genetically encoding functional moieties into its protein building blocks prior to assembly. To date, various functional versions of the ZT polymer have been produced that display bioactive peptidic motifs (Bruning et al., 2010; Hill et al., 2019) as well as functional full-length proteins introduced by gene fusion (Hill et al., 2019; Nesterenko et al., 2019). A ZT variant comprising the integrin-binding $\mathrm{Fn}^{10}$ domain from human $\mathrm{Fn}\left(\mathrm{ZT}^{\mathrm{Fn} 10}\right)$ has been shown to successfully sustain the culturing of human embryonic stem cells (hESCs), murine mesenchymal stromal cells (mMSCs) (Hill et al., 2019), and human induced pluripotent stem cells (hiPSCs) (Nesterenko et al., 2019) over extended periods of time. Its robustness, the ease of its handling as a biocoating, and the economy of its production, make the fibronectin-mimetic $\mathrm{ZT}^{\mathrm{Fn} 10}$ a promising system to replace native $\mathrm{Fn}$ in high-throughput cell applications. Previous applications of the ZT material had focused on its cell adhesion and culturing properties, while its ability to support naturalistic cell migration in functional assays has not been tested so far.

In this study, we investigate the suitability of the ZT system to support NCC culturing and migration in the cMINC assay. In particular, we study whether cell migration on this recombinant substrate displays traits comparable to those on the native ECM-component Fn and whether the migration provides sufficient sensitivity to support its application to the screening of toxicants in the cMINC assay.

Abbreviations

cMINC, circular migration inhibition of neural crest cells; CytoD, cytochalasin D; DMSO, dimethyl sulfoxide; ECM, extracellular matrix; FBS, fetal bovine serum; Fn, fibronectin; iPSC, induced pluripotent stem cells; NCC, neural crest cell; PCB, polychlorinated biphenyl; Tel, telethonin; TEV, tobacco etch virus; ZT, $Z_{1212} /$ telethonin polymer 


\section{Materials and methods}

\section{Protein production}

Expression clones of telethonin (Tel), $\mathrm{Z}_{1212}$, and $\mathrm{Z}_{1212}{ }^{\mathrm{Fn} 10}$ have been previously reported (Hill et al., 2019; Bruning et al., 2010). In brief, all coding sequences were inserted into the pETM-11 vector (EMBL), which adds a His 6 -tag and a tobacco etch virus (TEV) protease cleavage site N-terminally to the inserted gene.

Proteins were expressed in BL21 E. coli cells (Invitrogen) grown at $37^{\circ} \mathrm{C}$ in Luria Bertani (LB) medium supplemented with $25 \mu \mathrm{g} / \mathrm{mL}$ kanamycin. At a culture $\mathrm{OD}_{600}=0.6$, protein expression was induced with $1 \mathrm{mM}$ IPTG, the incubation temperature was reduced to $18^{\circ} \mathrm{C}$, and expression was carried out for $16-18 \mathrm{~h}$ overnight. Cells were harvested by centrifugation. Cell pellets with overexpressed $Z_{1212}, Z_{1212}{ }^{\text {Fn10 }}$ and Tel proteins were resuspended in $50 \mathrm{mM}$ Tris- $\mathrm{HCl}, 500 \mathrm{mM} \mathrm{NaCl}, \mathrm{pH} 7.4$ supplemented with an ethylenediaminetetraacetic acid (EDTA)-free protease inhibitor cocktail (Roche). Cells were lysed by sonication. Lysates were clarified by centrifugal ultrafiltration and filtered $(0.2 \mu \mathrm{m}$ pore filter; Sarstedt) prior to sample purification. $Z_{1212}$ and $\mathrm{Z}_{1212}{ }^{\mathrm{Fn} 10}$ were isolated from the soluble lysate fraction via $\mathrm{Ni}^{2+}$ IMAC ( 5 mL His-Trap HiPrep column; GE healthcare) in the buffer described above. The $\mathrm{His}_{6}$-tag was cleaved by incubation with TEV protease in dialysis overnight in $50 \mathrm{mM}$ Tris- $\mathrm{HCl} \mathrm{pH} \mathrm{7.4,}$ $500 \mathrm{mM} \mathrm{NaCl}$. The sample was then further purified by subtractive $\mathrm{Ni}^{2+}$-IMAC, followed by dialysis (in $50 \mathrm{mM}$ Tris- $\mathrm{HCl}, 100$ $\mathrm{mM} \mathrm{NaCl} \mathrm{pH} \mathrm{7.4)} \mathrm{and} \mathrm{gel} \mathrm{filtration} \mathrm{on} \mathrm{a} \mathrm{HiLoad} \mathrm{26/600} \mathrm{Superdex}$ 200 column (GE Healthcare) equilibrated in dialysis buffer.

Tel was purified from the insoluble lysis fraction as described previously (Hill et al., 2019). Briefly, the insoluble fraction was resuspended in $25 \mathrm{mM}$ Tris- $\mathrm{HCl}, 6 \mathrm{M}$ urea, $\mathrm{pH} 7.4$, lysed and centrifuged. The pellet was discarded and the supernatant was filtered and subsequently subjected to purification using $\mathrm{Ni}^{2+}$ IMAC. The Tel isolate was concentrated to $10 \mathrm{mg} / \mathrm{mL}$ in the buffer described above and stored at $6^{\circ} \mathrm{C}$.

\section{Production of the ZT ${ }^{\text {Fn10 }}$ polymer by assembly of $Z_{1212}{ }^{\text {Fn10 }}$ and Tel}

$\mathrm{Z}_{1212}, \mathrm{Z}_{1212}{ }^{\mathrm{Fn} 10}$ and Tel samples were filtered using $0.22 \mu \mathrm{m} \mathrm{Ul}$ trafree-MC filters (Merck), and all subsequent handling was carried out in a clean bench under laminar flow. For polymerization of the proteins to form the $\mathrm{ZT}$ and $\mathrm{ZT}^{\mathrm{Fn} 10}$ substrates, a protocol adapted from Bruning et al. (2010) was used. $Z_{1212}$ or $Z_{1212}$ Fn10 samples were mixed with Tel at a 1:3 molar ratio in overnight dialysis in $50 \mathrm{mM}$ Tris- $\mathrm{HCl}, 100 \mathrm{mM} \mathrm{NaCl}, \mathrm{pH} 7.4$ at room temperature and using a Slide-A-Lyzer ${ }^{\mathrm{TM}} \mathrm{MINI}$ dialysis device $3.5 \mathrm{~K}$ MWCO (Thermo Fisher Scientific) under sterile conditions. Samples were incubated for $24 \mathrm{~h}$ until self-assembly had led to a turbid sample pellet. The sample was then retrieved from the dialysis setting and stored at $6^{\circ} \mathrm{C}$ until further use. Self-assembly was confirmed by native polyacrylamide gel electrophoresis.

\section{Coating of polystyrene plates with substrata}

Bovine plasma fibronectin (Sigma), ZT and $\mathrm{ZT}^{\mathrm{Fn} 10}$ assemblies were diluted in low-endotoxin Dulbecco's phosphate buffered saline (DPBS) without $\mathrm{Ca}^{2+}$ and $\mathrm{Mg}^{2+}$ (Merck Millipore) to $1 \mu \mathrm{g}$ /
$\mathrm{mL}$ for bovine plasma fibronectin (Sigma) and to 1, 5, 10, $20 \mu \mathrm{g}$ / $\mathrm{mL}$ for $\mathrm{ZT}$ and $\mathrm{ZT}^{\mathrm{Fn} 10}$. Coating of 96-well polystyrene plates (Corning) was performed by deposition, which ensued by adding $100 \mu \mathrm{L}$ substrate solution to each well and incubating overnight at $37^{\circ} \mathrm{C}$. After $24 \mathrm{~h}$, solutions were removed by aspiration and wells dried by exposure to air for 15 min under a laminar flow.

\section{Neural crest cell differentiation}

NCCs were differentiated from the induced pluripotent stem cell (iPSC) line IMR90_clone_\#4 (WiCell, Wisconsin) following the modified protocol of Mica et al. (2013). IMR90 cells were maintained on human Laminin-521 (BioLamina) coating in essential 8 (E8) medium (DMEM/F12 supplemented with 15 mM HEPES buffer (Gibco), $16 \mathrm{mg} / \mathrm{mL}$ L-ascorbic-acid (Sigma-Aldrich), $0.7 \mathrm{mg} / \mathrm{mL}$ sodium selenite (Sigma-Aldrich), $20 \mu \mathrm{g} / \mathrm{mL}$ insulin (Sigma-Aldrich), $10 \mu \mathrm{g} / \mathrm{mL}$ holo-transferrin (Sigma-Aldrich), $100 \mathrm{ng} / \mathrm{mL}$ bFGF (Invitrogen), 1.74 ng/mL TGF $\beta$ (R\&D Systems)). For differentiation into NCCs, IMR90 iPSCs were plated on Matrigel $^{\mathrm{TM}}$ (Corning) coated plates at a density of 40,00050,000 cells $/ \mathrm{cm}^{2}$ in E8 medium containing $10 \mu \mathrm{M}$ ROCK-inhibitor (Y-27632 (Tocris). After two days, cells reached a confluency of $70-80 \%$, and differentiation was initiated (day 0 ) by a medium change to KSR medium (Knock out DMEM, 15\% knock out serum replacement, 1\% GlutaMax, 1\% MEM NEAA solution, $50 \mu \mathrm{M}$ 2-mercaptoethanol (all from Gibco)) supplemented with $20 \mathrm{ng} / \mathrm{mL}$ Noggin (R\&D Systems) and $10 \mu \mathrm{M} \mathrm{SB} 431542$ (Tocris). From day 2 on, cells were treated with $3 \mu \mathrm{M}$ CHIR 99021 (Axon Medchem). Noggin and SB431542 were withdrawn on days 3 and 4, respectively. Beginning on day 4, the KSR medium was gradually replaced with N2-S medium (DMEM/F12 (Gibco), $1.55 \mathrm{mg} / \mathrm{mL}$ glucose (Sigma-Aldrich), 1\% GlutaMax (Gibco), $0.1 \mathrm{mg} / \mathrm{mL}$ apotransferin (Sigma-Aldrich), $25 \mu \mathrm{g} / \mathrm{mL}$ insulin (Sigma-Aldrich), $20 \mathrm{nM}$ progesterone (Sigma-Aldrich), $100 \mu \mathrm{M}$ putrescine (Sigma-Aldrich), $30 \mathrm{nM}$ selenium (Sigma-Aldrich)) in $25 \%$ increments. Cells were collected on day 11 , resuspended in N2-S medium supplemented with $20 \mathrm{ng} / \mathrm{mL}$ epidermal growth factor (EGF) (R\&D Systems) and $20 \mathrm{ng} / \mathrm{mL}$ fibroblast growth factor 2 (FGF2) (R\&D Systems), and seeded as droplets $(10 \mu \mathrm{L})$ on poly-L-ornithine (PLO)/laminin/fibronectin (all from Sigma-Aldrich) coated $10 \mathrm{~cm}$ dishes. Cells were expanded by weekly splitting. From now on, seeding as droplets was not necessary and medium was changed every second day. After 35-39 days, cells were cryopreserved at a concentration of $4 \times 10^{6}$ cells $/ \mathrm{mL}$ in 90\% fetal bovine serum (FBS) (PAA Laboratories $\mathrm{GmbH}$ ) and $10 \%$ dimethyl sulfoxide (DMSO) (Merck Millipore) until further use. We found that cryopreservation of cells in serum-free medium (DMEM/F12, $1.55 \mathrm{mg} / \mathrm{mL}$ glucose, 1\% GlutaMax, $0.1 \mathrm{mg} /$ $\mathrm{mL}$ apotransferin, $25 \mu \mathrm{g} / \mathrm{mL}$ insulin, $20 \mathrm{nM}$ progesterone, 100 $\mu \mathrm{M}$ putrescine, $30 \mathrm{nM}$ selenium, 10\% DMSO) also worked, and this procedure will be used for future studies (data not shown).

\section{Migration assay (cMINC)}

The cMINC assay was performed as described earlier (Nyffeler et al., 2017b). Briefly, silicone stoppers (Platypus Technologies, Madison, WI, US) were placed centrally into each experimental well of a 96-well polystyrene plate (Corning) coat- 
ed with plasma fibronectin, $\mathrm{ZT}$ or $\mathrm{ZT}^{\mathrm{Fn} 10}$. Cells were seeded into the stopper-containing wells at a density of 95,000 cells/ $\mathrm{cm}^{2}$. The following day, stoppers were removed to allow cells to migrate into the cell-free central area, and medium was refreshed. To test the effect of toxicants on NCC motility, 5x concentrated toxicant (cytochalasin D (CytoD), lithium chloride (LiCl), and polychlorinated biphenyl 180 (PCB180); (all from Sigma-Aldrich)) solution was added to the medium $24 \mathrm{~h}$ after stopper removal. After another $24 \mathrm{~h}$, cell viability and migration endpoints were monitored. For this, cells were stained with Hoechst-33342 and calcein-AM (both from SigmaAldrich), and image acquisition was performed using a Cellomics ArrayScan VTI imaging microscope (Thermo Fisher). Hoechst33342 and calcein-AM double-positive cells were determined by an automated algorithm described earlier (Stiegler et al., 2011; Krug et al., 2013) and defined as viable cells. For migration quantification, a free software too ${ }^{2}$ was used as described in Nyffeler et al. (2017b) to calculate the original stopper position and determine the number of viable cells within the migration area. Viability and migration were normalized to untreated or solvent control (0.1\% DMSO). Concentration-response curves were generated with GraphPad Prism (GraphPad Software, La Jolla, CA, USA); each data point represents mean \pm SEM of three biological replicates. For curve fitting, a four-parameter log-logistic curve fit with an upper asymptote forced to $100 \%$ was used.

\section{Time-lapse experiment and cell tracking}

The cMINC assay was performed using an Axio Observer Z1 microscope (Zeiss, Germany) equipped with an Axiocam MRM camera to track cell movement into the central "migration zone" for $24 \mathrm{~h}$ after stopper removal. Phase contrast images were acquired every $10 \mathrm{~min}$ with a $5 \mathrm{x}$ objective. To provide constant temperature and $\mathrm{CO}_{2}$ supply during the recording time, a microscope equipped with an incubation system was used. Cell tracking was performed manually using the "Manual Tracking" plugin in ImageJ (Schneider et al., 2012) by selecting, in reverse time, viable cells that migrated into the central area and were visible throughout the full time-lapse recording. A total of 60 individual cells were tracked for each condition (namely, per media condition and concentration, three technical replicates were performed in two biological replicates, with 10 cells tracked per technical replicate). To determine cell distance and velocity, cell location coordinates on the viewed plate were obtained with ImageJ (Schneider et al., 2012) and transferred to the "Chemotaxis and Migration Tool V2.0" (ibidi, Germany). Cell translocation, accumulated distance, directness and cell speed parameters were calculated from the coordinates of the migrating cells. These parameters were defined as follows: i) Cell translocation refers to the straight line formed by connecting the start and end points of the migration path of the cell. ii) Accumulated distance is the sum of incremental movements made by the cell during migration from the start to the end point. iii) Directness is the ratio between cell translocation and accumulated distance, where the closer the value is to 1 , the more directional the migration is. iv) Cell speed is the ratio between the accumulated distance and the time length of the images acquired during cell migration.

\section{Data handling and statistics}

If not stated otherwise, values are expressed as mean \pm SEM. If not indicated otherwise, experiments were performed at least three times (i.e., using three different cell preparations), with at least three technical replicates per condition.

\section{Results and discussion}

\subsection{Migration of neural crest cells on the ZTFn 10 polymer}

In order to investigate whether the $\mathrm{ZT}^{\mathrm{Fn} 10}$ biomaterial may substitute the established bovine serum Fn coating in the cMINC assay setup, we compared the migration of NCCs on the novel ZT matrix to that on the classical coating. Two variants of the ZT polymer were compared in these experiments: the integrininteracting $\mathrm{ZT}^{\mathrm{Fn} 10}$ form and the non-functionalized ZT scaffold, known to be bioinert (Hill et al., 2019). The latter served as control to reveal any possible non-specific interactions between NCCs and the ZT core structure.

First, we evaluated the viability and retention of NCCs on the ZT substrates. For this, the polymers were passively adsorbed onto polystyrene 96-well cell culture plates, and NCCs were plated on the coated surfaces. The number of viable NCCs remaining on the plates was assessed $72 \mathrm{~h}$ after seeding. As positive control, the cell count on the classical Fn-coating $(1 \mu \mathrm{g} / \mathrm{mL})$ was evaluated. Non-coated dishes were also assessed. The results revealed that NCCs adhered about equally well to plates under all test conditions, including non-coated plates (Fig. 1A). This revealed that cell attachment was not dependent on a specific ECM component. This situation allowed us to start migration with similar numbers of cells on the different matrices.

In a second step, NCC migration capacity was monitored using the cMINC assay (Nyffeler et al., 2017b). Here, a clear dependence of migration on the type of coating was observed (Fig. 1b,c) (Fig. S1 ${ }^{3}$, movies MS1-MS44). Cells cultured on the classical bovine Fn migrated towards the center of the well, while cells cultured on non-coated polystyrene migrated only to a small extent. The number of cells migrating on the ZT control material was comparable to the number of cells migrating on the non-coated polystyrene, confirming its bioinert character. In contrast, cells seeded on $\mathrm{ZT}^{\mathrm{Fn} 10}$ migrated quickly and with good directionality.

\footnotetext{
2 http://invitrotox.uni-konstanz.de/

3 doi:10.14573/altex.2003181s1

4 doi:10.14573/altex.2003181s2; doi:10.14573/altex.2003181s3; doi:10.14573/altex.2003181s4; doi:10.14573/altex.2003181s5
} 
A
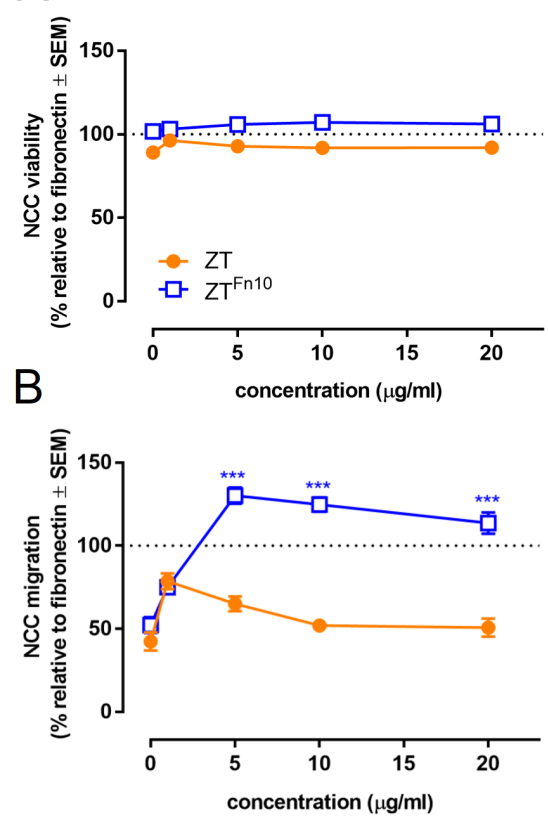

C
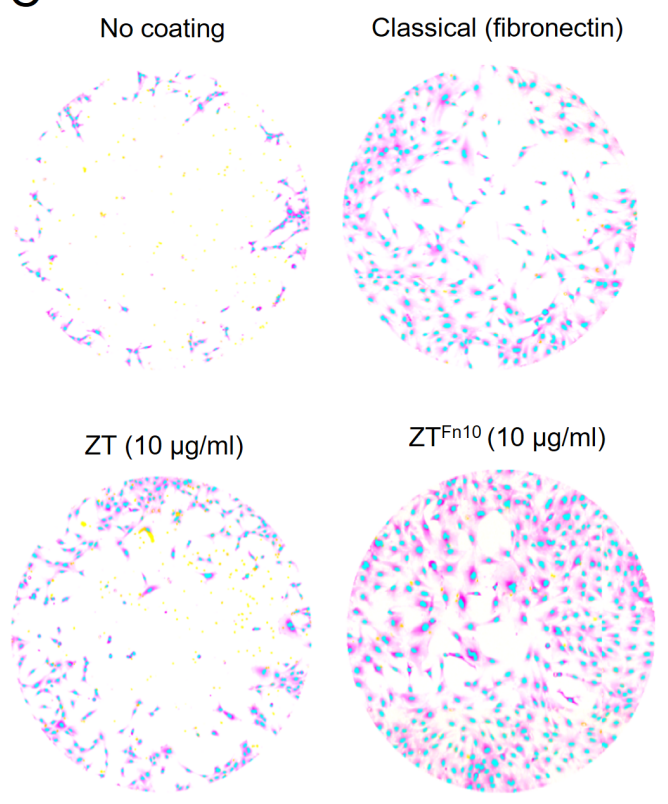

Fig. 1: Neural crest cell viability and migration

(A) Neural crest cell viability at $72 \mathrm{~h}$ after seeding on the non-functionalized (inert) ZT polymer ( $\mathrm{n}=30$; data points obtained from three cell preparations, each with 10 technical replicates) and on $\mathrm{ZT}^{\mathrm{Fn} 10}(\mathrm{n}=15$; three cell preparations with five technical replicates each). (B) Neural crest cell migration at $72 \mathrm{~h}$ after seeding on $\mathrm{ZTFn10}$ and on $\mathrm{ZT}$. In both cases, data are shown as means from three cell preparations. ${ }^{* *}, \mathrm{p}<0.001$ as determined by two-way ANOVA (considering type of coating as factor) followed by Bonferroni's post-hoc test. (C) Exemplary fluorescence images of NCCs stained with Hoechst-33342 (nuclei, cyan) and calcein-AM (cytosol, magenta) at the end of migration experiments on diverse coatings (as indicated). The diameter of each of the circular areas displayed was $2 \mathrm{~mm}$.

During the development of the cMINC assay, the Fn-coating had been tested in detail. Maximal migration was observed at a concentration of approx. $5 \mu \mathrm{g} / \mathrm{mL}$ (Fig. S2 $\mathrm{A}^{3}$ ); however, $1 \mu \mathrm{g} / \mathrm{mL}$ was found to be the optimal balance between assay performance and costs (Nyffeler et al., 2017b), and this condition was then standardized by deposition of the assay protocol in public repositories (Krebs et al., 2019) and inclusion of the test in international screening programs (Bal-Price et al., 2018). Here, we retested a wide concentration range for comparison to the new substrate. Our data confirmed $1 \mu \mathrm{g} / \mathrm{mL}$ Fn as a suitable and robust standard condition (little variability, good assay window) (Fig. S2 $\mathrm{C}^{3}$ ). At low concentrations of Fn, migration was continuously improved by increasing the concentration of the coating material until a maximum was reached. At very high concentrations, migration was attenuated as cells tended to attach too strongly to move.

The current work shows that the optimal ZT $\mathrm{T}^{\mathrm{Fn} 10}$ substrate concentration for NCC migration is also $5 \mu \mathrm{g} / \mathrm{mL}$. This is in good agreement with data from a previous study that found that saturation of stem cell attachment was attained at a substrate concentration of around 5-10 $\mu \mathrm{g} / \mathrm{mL}$ (Hill et al., 2019). Importantly, cost and availability are not limiting for $\mathrm{ZT}^{\mathrm{Fn} 10}$, so that its implementation in high-throughput assays at this optimal concentration is realistic. At even higher concentrations we observed a trend to reduced migration, in good accordance with a bell-shaped migration behavior on ECM (Underwood et al., 1993).

In summary, $\mathrm{ZT}^{\mathrm{Fn} 10}$ allowed optimal migration that was fully comparable to Fn. The optimum concentration was $5 \mu \mathrm{g} / \mathrm{mL}$, and at this concentration, the migration performance was even better than at standard assay conditions, i.e., $1 \mu \mathrm{g} / \mathrm{mL} \mathrm{Fn}$.

\subsection{Examination of migration speed and directionality at the single cell level}

To characterize the migration behavior of NCCs on $\mathrm{ZT}^{\mathrm{Fn} 10}$ in greater detail, the cMINC assay setup was used for single cell tracking. For this, cells were seeded onto coated plates as described above and migration was monitored by time-lapse video microscopy for $24 \mathrm{~h}$ at $10 \mathrm{~min}$ intervals. Different features of the recorded movement trajectories were quantified. These data fully confirmed the observations with the classical cMINC read-out: Cells seeded on optimal concentrations of $\mathrm{ZT}^{\mathrm{Fn} 10}$ migrated at higher speed, cov- 


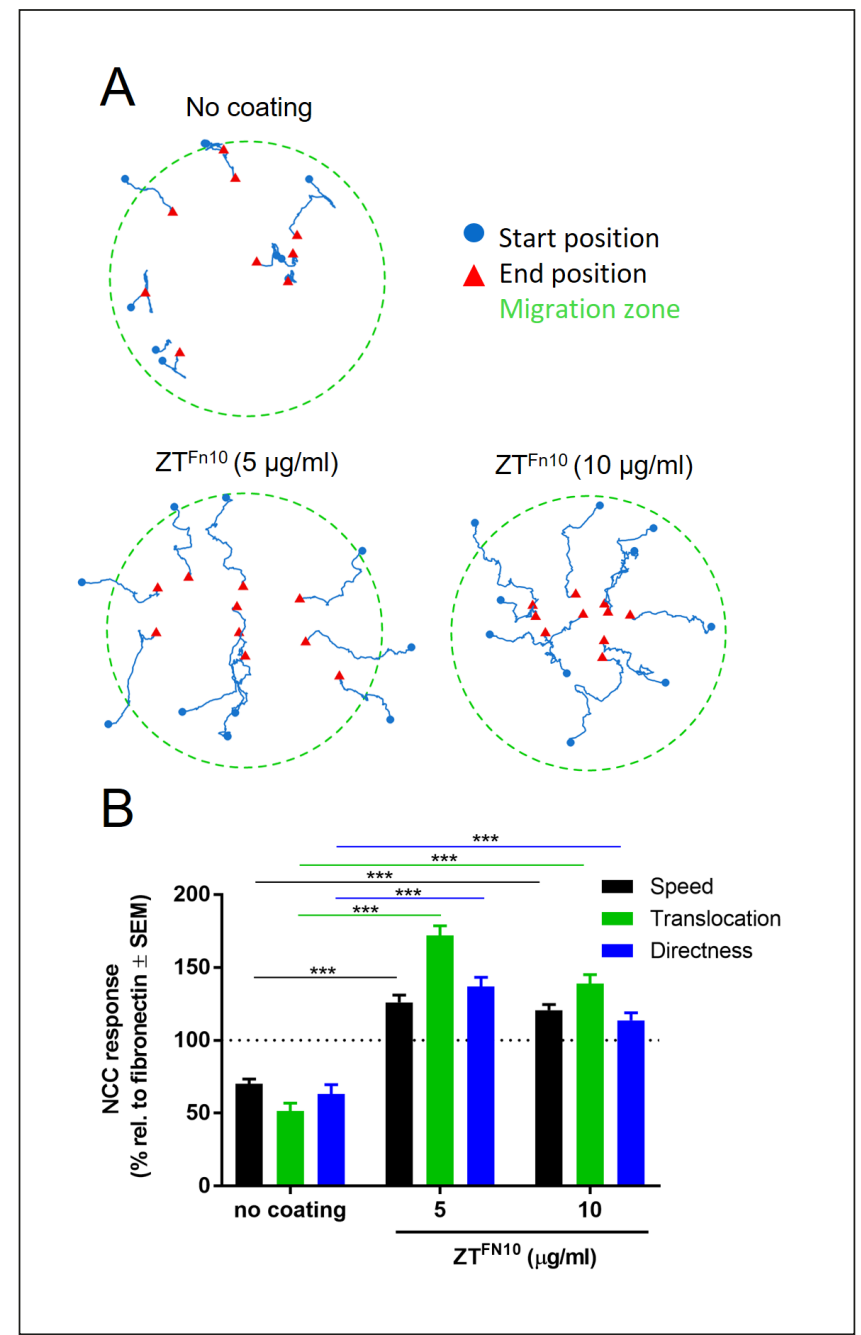

Fig. 2: Analysis of migration trajectories of neural crest cells NCCs were plated around silicone stoppers and allowed to attach for $24 \mathrm{~h}$. The stoppers were removed, and NCC migration was observed for $24 \mathrm{~h}$ using time-lapse imaging. The tracks were marked in the ImageJ program and evaluated manually. (A) Representative trajectories of neural crest cells upon migration into an initially empty circular area (delineated by green circles). (B) Speed (distance along the track per time), cell translocation (Euclidean distance from start to endpoint of track) and directness (cell translocation/track length, i.e., "low degree of zig-zagging"), each calculated from the trajectory of 20 cells from two cell preparations. ${ }^{* * *}, p<0.001$ as determined by ANOVA (considering type of coating as factor; performed separately for each endpoint) followed by Dunnett's post-hoc test.

ered longer distances, and moved in a more directed fashion than those in the classical cMINC setup (Fig. 2). However, as $\mathrm{ZT}^{\mathrm{Fn} 10}$ is a fibronectin mimetic, the expectation was that when using both substrates at their optimal concentrations $(5 \mu \mathrm{g} / \mathrm{mL}$ in both cases), the migration would be similar on both substrates. Effectively, this was confirmed experimentally (Fig. S2B ${ }^{3}$ ), proving that NCC migration was equivalent on both substrates.
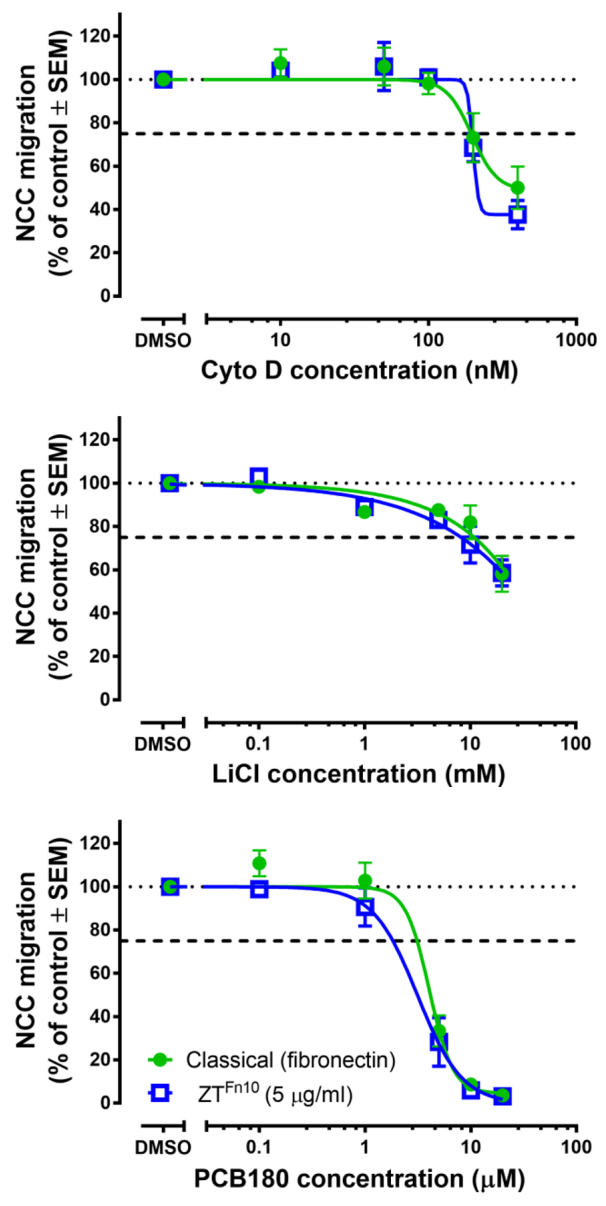

Fig. 3: Migration inhibition of NCCs by model toxicants

NCCs were plated under otherwise similar conditions on dishes coated with fibronectin or $\mathrm{ZT}^{\mathrm{Fn} 10}$. They were exposed to cytochalasin D (CytoD), lithium chloride ( $\mathrm{LiCl}$ ) and polychlorinated biphenyl 180 (PCB180) for $24 \mathrm{~h}$. Initial values (100\%) correspond to untreated cells (control cell medium containing $0.1 \%$ DMSO as "negative control"). Cell migration data are all given relative to negative control values. Each data point shown is the mean \pm SEM of three biological replicates (= different cell preparations). Dashed lines at $75 \%$ visualize the threshold value for impaired cell migration.

\subsection{Comparative testing of test method positive controls on classical and novel coatings}

As the $\mathrm{ZT}^{\mathrm{Fn} 10}$ substrate was shown to support NCC migration, we tested its suitability as a substitute of plasma Fn in the cMINC toxicity test. For this, we ran parallel tests on $\mathrm{ZT}^{\mathrm{Fn} 10}$ and Fn. As model toxicants, we used the well-established NCC migration inhibitors cytochalasin D (CytoD), lithium chloride ( $\mathrm{LiCl})$, and 
polychlorinated biphenyl 180 (PCB180) (Fig. 3). The selected toxicants have largely different chemical structures and modes of action. They also act over a wide range of concentrations (100 $\mathrm{nM}-10 \mathrm{mM})$. Most importantly, they are all known to affect migration at lower concentrations than cell viability. This was fully confirmed here (see Fig. $S 3^{3}$ for viability data). The results showed that concentration-response curves for impaired migration were similar for the novel $\mathrm{ZT}^{\mathrm{Fn} 10}$ coating material and for native Fn. We conclude that the $\mathrm{ZT}^{\mathrm{Fn} 10}$ substrate can be used instead of Fn for further toxicological applications of the cMINC assay.

\section{Conclusions and outlook}

This study was undertaken to examine the feasibility of exchanging animal-derived cell culture supplements for defined bioengineered recombinant material. The work exemplifies the type of bridging studies required to change established assay protocols. In some cases, assay conditions may need adaptations to new materials and assay performance might require re-examination. Here, the requirement for adaptations was minimal, with the new material being readily applicable to the toxicology application under consideration. Notably, the ECM-native Fn and the ZT ${ }^{\mathrm{Fn} 10}$ material proved to be applicable in similar concentration ranges. It is noteworthy that the concentration affected only a basic test system parameter (number of migrating cells under control conditions) but not the assay performance under toxicant challenge. These results suggest that ECM-substitute materials hold high promise in high-throughput toxicology applications and now encourage the exploration of these materials in other tests, e.g., chemotaxis assays for T-cells and neutrophils (Lin and Butcher, 2006; Cano et al., 2016). In this regard, it is worth emphasizing that the $\mathrm{ZT}^{\mathrm{Fn} 10}$ material has demonstrated excellent cell compatibility and that its sole protein composition, self-assembly and molecular domain granularity is reminiscent of that of typical biological matrices. Moreover, its modular construction readily allows the incorporation of alternative cell attachment and functional domains by genetic modification, as exemplified by other available $\mathrm{ZT}$ variants that carry fluorescent proteins and functional peptides (Nesterenko et al., 2019). In summary, our findings suggest that the replacement of animal-sourced cell substrates in in vitro toxicology screenings now appears feasible thanks to recent biomaterial advances.

\section{Material availability}

The ZT ${ }^{\mathrm{Fn} 10}$ substrate can be obtained from the authors (olga. mayans@uni-konstanz.de).

\section{References}

Bal-Price, A., Hogberg, H. T., Crofton, K. M. et al. (2018). Recommendation on test readiness criteria for new approach methods in toxicology: Exemplified for developmental neurotoxicity. ALTEX 35, 306-352. doi:10.14573/altex.1712081

Bergeron, K. F., Cardinal, T. and Pilon, N. (2013). A quantitative cell migration assay for murine enteric neural progenitors. $J$ Vis Exp, e50709. doi:10.3791/50709

Bruning, M., Kreplak, L., Leopoldseder, S. et al. (2010). Bipartite design of a self-fibrillating protein copolymer with nanopatterned peptide display capabilities. Nano Lett 10, 4533-4537. doi:10.1021/nl1024886

Cai, L. and Heilshorn, S. C. (2014). Designing ECM-mimetic materials using protein engineering. Acta Biomater 10, 17511760. doi:10.1016/j.actbio.2013.12.028

Cano, P. M., Vargas, A. and Lavoie, J. P. (2016). A real-time assay for neutrophil chemotaxis. Biotechniques 60, 245-251. doi:10.2144/000114416

Chi-Rosso, G., Gotwals, P. J., Yang, J. et al. (1997). Fibronectin type III repeats mediate RGD-independent adhesion and signaling through activated beta1 integrins. J Biol Chem 272, 31447-31452. doi:10.1074/jbc.272.50.31447

Conway, J. R. W. and Jacquemet, G. (2019). Cell matrix adhesion in cell migration. Essays Biochem 63, 535-551. doi:10.1042/ ebc20190012

Farlie, P. G., McKeown, S. J. and Newgreen, D. F. (2004). The neural crest: Basic biology and clinical relationships in the craniofacial and enteric nervous systems. Birth Defects Res $C$ Embryo Today 72, 173-189. doi:10.1002/bdrc.20013

Fuller, L. C., Cornelius, S. K., Murphy, C. W. et al. (2002). Neural crest cell motility in valproic acid. Reprod Toxicol 16, 825839. doi:10.1016/s0890-6238(02)00059-x

Hackland, J. O. S., Frith, T. J. R., Thompson, O. et al. (2017). Top-down inhibition of BMP signaling enables robust induction of hPSCs into neural crest in fully defined, xeno-free conditions. Stem Cell Reports 9, 1043-1052. doi:10.1016/j.stemcr. 2017.08.008

Hellmund, K. S. and Koksch, B. (2019). Self-assembling peptides as extracellular matrix mimics to influence stem cell's fate. Front Chem 7, 172. doi:10.3389/fchem.2019.00172

Henderson, D. J. and Copp, A. J. (1997). Role of the extracellular matrix in neural crest cell migration. J Anat 191, 507-515. doi:10.1046/j.1469-7580.1997.19140507.x

Hill, C. J., Fleming, J. R., Mousavinejad, M. et al. (2019). Selfassembling proteins as high-performance substrates for embryonic stem cell self-renewal. Adv Mater 31, e1807521. doi: 10.1002/adma.201807521

Hughes, C. S., Postovit, L. M. and Lajoie, G. A. (2010). Matrigel: A complex protein mixture required for optimal growth of cell culture. Proteomics 10, 1886-1890. doi:10.1002/pmic. 200900758

Krebs, A., Waldmann, T., Wilks, M. F. et al. (2019). Template for the description of cell-based toxicological test methods to allow evaluation and regulatory use of the data. ALTEX 36, 682699. doi:10.14573/altex.1909271

Krug, A. K., Balmer, N. V., Matt, F. et al. (2013). Evaluation of a human neurite growth assay as specific screen for developmental neurotoxicants. Arch Toxicol 87, 2215-2231. doi:10.1007/ s00204-013-1072-y

LaBonne, C. and Bronner-Fraser, M. (1999). Molecular mechanisms of neural crest formation. Annu Rev Cell Dev Biol 15, 81-112. doi:10.1146/annurev.cellbio.15.1.81 
Lin, F. and Butcher, E. C. (2006). T cell chemotaxis in a simple microfluidic device. Lab Chip 6, 1462-1469. doi:10.1039/ b607071j

Loo, Y., Goktas, M., Tekinay, A. B. et al. (2015). Self-assembled proteins and peptides as scaffolds for tissue regeneration. $A d v$ Healthc Mater 4, 2557-2586. doi:10.1002/adhm.201500402

Marino, M., Zou, P., Svergun, D. et al. (2006). The Ig doublet Z1Z2: A model system for the hybrid analysis of conformational dynamics in Ig tandems from titin. Structure 14, 14371447. doi:10.1016/j.str.2006.07.009

Mica, Y., Lee, G., Chambers, S. M. et al. (2013). Modeling neural crest induction, melanocyte specification, and diseaserelated pigmentation defects in hESCs and patient-specific iPSCs. Cell Rep 3, 1140-1152. doi:10.1016/j.celrep.2013.03. 025

Nesterenko, Y., Hill, C. J., Fleming, J. R. et al. (2019). The ZT biopolymer: A self-assembling protein scaffold for stem cell applications. Int J Mol Sci 20, 4299. doi:10.3390/ ijms20174299

Nyffeler, J., Dolde, X., Krebs, A. et al. (2017a). Combination of multiple neural crest migration assays to identify environmental toxicants from a proof-of-concept chemical library. Arch Toxicol 91, 3613-3632. doi:10.1007/s00204-017-1977-y

Nyffeler, J., Karreman, C., Leisner, H. et al. (2017b). Design of a high-throughput human neural crest cell migration assay to indicate potential developmental toxicants. ALTEX 34, 75-94. doi:10.14573/altex.1605031

Oredsson, S., Coecke, S., van der Valk, J. et al. (2019). What is understood by "animal-free research"? Toxicol In Vitro 57, 143-144. doi:10.1016/j.tiv.2019.03.001

Pankov, R. and Yamada, K. M. (2002). Fibronectin at a glance. $J$ Cell Sci 115, 3861-3863. doi:10.1242/jcs.00059

Perris, R., Paulsson, M. and Bronner-Fraser, M. (1989). Molecular mechanisms of avian neural crest cell migration on fibronectin and laminin. Dev Biol 136, 222-238. doi:10.1016/00121606(89)90144-9

Reichman, S., Slembrouck, A., Gagliardi, G. et al. (2017). Generation of storable retinal organoids and retinal pigmented epithelium from adherent human iPS cells in xeno-free and feeder-free conditions. Stem Cells 35, 1176-1188. doi:10.1002/stem.2586

Ridley, A. J., Schwartz, M. A., Burridge, K. et al. (2003). Cell migration: Integrating signals from front to back. Science 302, 1704-1709. doi:10.1126/science. 1092053

Schneider, C. A., Rasband, W. S. and Eliceiri, K. W. (2012). NIH image to ImageJ: 25 years of image analysis. Nat Methods 9 , 671-675. doi:10.1038/nmeth.2089

Scholz, D., Poltl, D., Genewsky, A. et al. (2011). Rapid, complete and large-scale generation of post-mitotic neurons from the human LUHMES cell line. J Neurochem 119, 957-971. doi:10.1111/j.1471-4159.2011.07255.x

Shafqat-Abbasi, H., Kowalewski, J. M., Kiss, A. et al. (2016). An analysis toolbox to explore mesenchymal migration heterogeneity reveals adaptive switching between distinct modes. Elife 5, e11384. doi:10.7554/eLife. 11384

Stiegler, N. V., Krug, A. K., Matt, F. et al. (2011). Assessment of chemical-induced impairment of human neurite outgrowth by multiparametric live cell imaging in high-density cultures. Toxicol Sci 121, 73-87. doi:10.1093/toxsci/kfr034

Sung, T. C., Liu, C. H., Huang, W. L. et al. (2019). Efficient differentiation of human ES and iPS cells into cardiomyocytes on biomaterials under xeno-free conditions. Biomater Sci 7, 5467-5481. doi:10.1039/c9bm00817a

Testaz, S., Delannet, M. and Duband, J. (1999). Adhesion and migration of avian neural crest cells on fibronectin require the cooperating activities of multiple integrins of the (beta) 1 and (beta)3 families. J Cell Sci 112, 4715-4728.

Underwood, P. A., Steele, J. G. and Dalton, B. A. (1993). Effects of polystyrene surface chemistry on the biological activity of solid phase fibronectin and vitronectin, analysed with monoclonal antibodies. J Cell Sci 104, 793-803.

Usami, M., Mitsunaga, K., Miyajima, A. et al. (2016). Effects of 13 developmentally toxic chemicals on the migration of rat cephalic neural crest cells in vitro. Congenit Anom (Kyoto) 56, 52-59. doi:10.1111/cga.12121

van der Valk, J., Bieback, K., Buta, C. et al. (2018). Fetal bovine serum (FBS): Past - Present - Future. ALTEX 35, 99-118. doi:10.14573/altex.1705101

Zimmer, B., Lee, G., Balmer, N. V. et al. (2012). Evaluation of developmental toxicants and signaling pathways in a functional test based on the migration of human neural crest cells. Environ Health Perspect 120, 1116-1122. doi:10.1289/ ehp.1104489

Zou, P., Pinotsis, N., Lange, S. et al. (2006). Palindromic assembly of the giant muscle protein titin in the sarcomeric Z-disk. Nature 439, 229-233. doi:10.1038/nature04343

\section{Conflict of interest}

The authors declare no conflict of interest.

\section{Acknowledgements}

This work was supported by the BMBF, EFSA, the DK-EPA (MST-667-00205), and the DFG (Konstanz Research School of Chemical Biology; KoRS-CB). It has received funding from the European Union's Horizon 2020 research and innovation programme under grant agreements No. 681002 (EU-ToxRisk) and No. 825759 (ENDpoiNTs). 